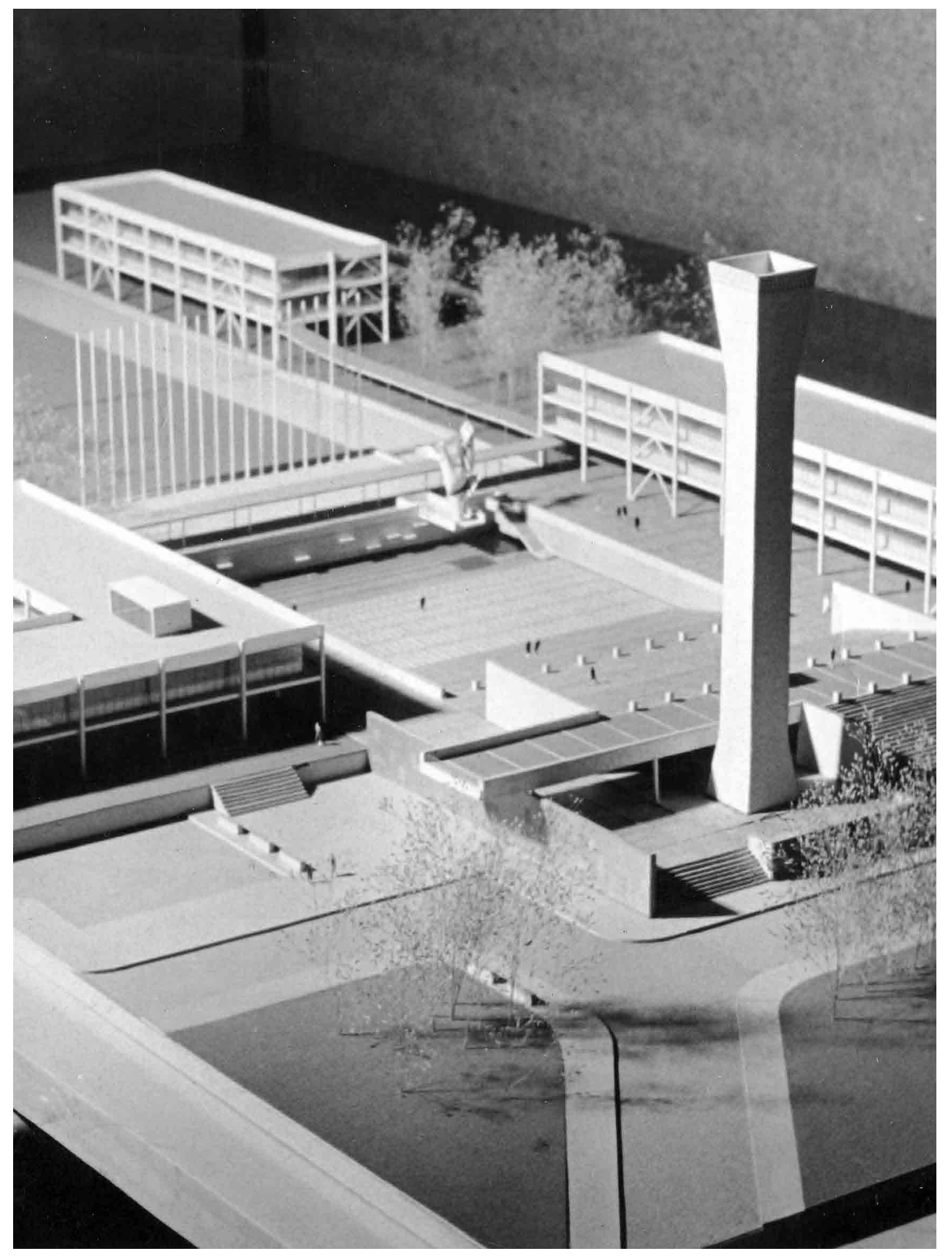

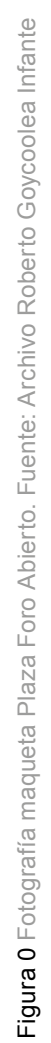



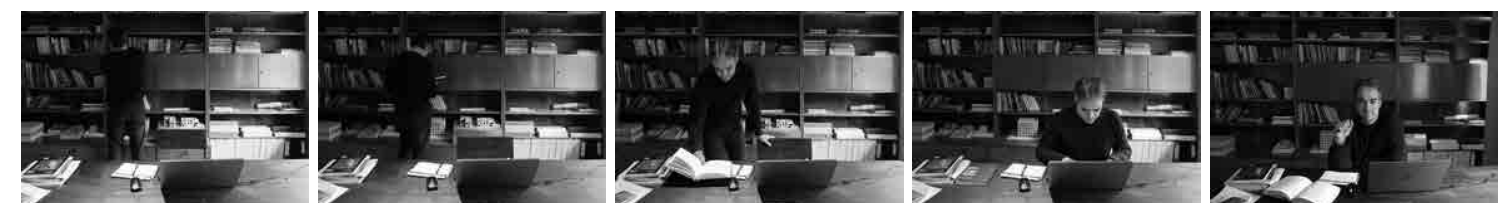

Fotos: Cristián Berríos

\title{
EMILIO DUHART, LA ARQUITECTURA COMO ESTRUCTURA DEL ESPACIO PÚBLICO: PLAZA FORO ABIERTO - UNIVERSIDAD DE CONCEPCIÓN' ${ }^{1}$
}

\author{
EMILIO DUHART, ARCHITECTURE AS THE STRUCTURE OF PUBLIC SPACE: PLAZA FORO \\ ABIERTO (OPEN FORUM SQUARE) - UNIVERSITY OF CONCEPCIÓN'
}

\section{Cristián Berríos Flores²}

\author{
RESUMEN
}

En el año 1957, Emilio Duhart Harosteguy recibe el encargo de planificar el crecimiento físico de la Universidad de Concepción para los siguientes 25 años. Lo que en un principio sería el desarrollo de un Plan Director del conjunto universitario, se transformó en una elaborada planificación urbano-arquitectónica, desde que al requerimiento se incorpora también la redacción de los proyectos de arquitectura. Aquello supone una doble oportunidad para Duhart: la de dotar de una estructura coherente a la totalidad de la organización urbana de la ciudad universitaria y la de dar forma al espacio público del conjunto mediante la precisa configuración definida por la nueva arquitectura. Este escrito intenta develar los criterios de proyecto convocados por el arquitecto para la conformación de la Plaza del Foro Abierto, espacio público que puede ser considerado como la síntesis de los argumentos elaborados por Duhart para la planificación general. Dicha plaza, que el mismo arquitecto señalaba como el centro de gravedad o corazón de la universidad, se ha transformado, a sesenta años de su génesis, en uno de los lugares públicos con mayor sentido de pertenencia e identidad para la ciudad de Concepción; premisa a partir de la cual se desarrolla este artículo.

Palabras clave: arquitectura, urbanismo, espacio público, ciudades universitarias, sistemas modulares.

\section{ABSTRACT}

In 1957, Emilio Duhart Harosteguy was commissioned to plan the physical growth of the University of Concepción for the next 25 years. What would initially be the development of a Master Plan for the entire university, became an elaborate urban-architectural plan, since the commission also included the drawing up of the associated architectural projects. This entailed a double opportunity for Duhart: to provide a coherent structure to the entire urban organization of the university campus and to shape the public space of the whole by means of the precise configuration defined by the new architecture. This paper attempts to reveal the project criteria assembled by the architect for the conformation of the Plaza del Foro Abierto (Open Forum Square), a public space that can be considered as the synthesis of the arguments elaborated by Duhart for the general plan. This square, which the architect himself designated as the center of gravity or heart of the university, has become, sixty years after its genesis, one of the public places with the greatest sense of belonging and identity for the city of Concepcion - the premise upon which this article is based.

Keywords: architecture, urban planning, public space, university campuses, modular systems.

Artículo recibido el 18 de enero de 2017 y aceptado el 11 de junio de 2017 DOI: https://doi.org/10.22320/07196466.2017.35.051.07

[1] Este artículo se basa en los resultados de investigación de la tesis doctoral desarrollada por Cristián Berríos Flores, "Emilio Duhart: Ciudad Universitaria de Concepción. Elaboración de un espacio urbano moderno", Universidad Politécnica de Cataluña, Barcelona, 2012.

[2] Depto. Diseño y Teoría de la Arquitectura, Universidad del Bío-Bío, Concepción, Chile. cberrios@ubiobio.cl 
En agosto de 1957, Emilio Duhart presentó a los directivos de la Universidad de Concepción un plano rotulado como "Esquema básico" (Figura 1). En este incipiente plano, que consideraba la ordenación general de la universidad, se puede apreciar de qué manera el arquitecto integra las preexistencias geográficas y urbanas del conjunto que habían sido planificadas por el destacado arquitecto austriaco, Karl Brunner, en 1931. Se evidencia la voluntad de expandir y dar continuidad a la organización física de la universidad por medio de dos ejes perpendiculares: hacia el sureste, Duhart daba profundidad al eje central promovido por Brunner, despuntando el cerro de un sector; hacia el noreste, avanzando en el ajustado valle, Duhart disponía de un conjunto de edificios paralelos, en el mismo sentido del eje sureste. En el encuentro de ambos ejes el arquitecto ubica una plaza que incorporaba al Campanil (1943), importante símbolo de la universidad.

En octubre de 1957, Duhart recibe el plano topográfico y los estudios de mecánica de suelos del conjunto, solicitados en mayo del mismo año. Con los informes de la calidad de los suelos, que confirmaban su mala calidad, Emilio Duhart sugiere al directorio que los futuros edificios de la universidad debían ser construidos en base a estructuras de acero, haciendo notar las cualidades de este sistema constructivo: menor peso sobre las fundaciones; organización flexible de recintos; industrialización de las etapas constructivas; y una posible colaboración con la vecina Compañía de Aceros del Pacífico ${ }^{3}$.

La recomendación de parte del arquitecto encuentra asidero en los miembros del directorio, quienes deciden sumar al encargo de la planificación de la universidad, también el desarrollo de los proyectos de arquitectura del futuro conjunto universitario. La integración de la elaboración de la arquitectura a la propuesta urbana por parte de Duhart, lo enfrenta a una doble oportunidad: dotar de una estructura urbana coherente a la planificación general de la universidad y dar forma al espacio público del conjunto mediante el manejo deliberado de los proyectos de arquitectura.

Develar el repertorio de criterios proyectuales que convoca Emilio Duhart en aras de configurar el espacio público de su propuesta para la Universidad de Concepción, es el propósito de este escrito. Cuando recibe este encargo, Duhart tiene 40 años y poseía ya una importante trayectoria profesional en proyectos de mediana y gran escala ${ }^{4}$. Asimismo, había llevado a cabo una insistente exploración académica sobre la relación de la arquitectura y el proyecto urbano, por lo cual se puede suponer que deposita aquí toda su experiencia práctica e implícitamente teórica. A modo de acotar el análisis sobre el caso de estudio, este artículo centra la mirada en la Plaza del Foro Abierto, a partir de la premisa de que el arquitecto dedica a tal espacio una especial atención, y de que efectivamente puede ser entendido como un proyecto de síntesis de su experiencia respecto a la elaboración de centros cívicos. 


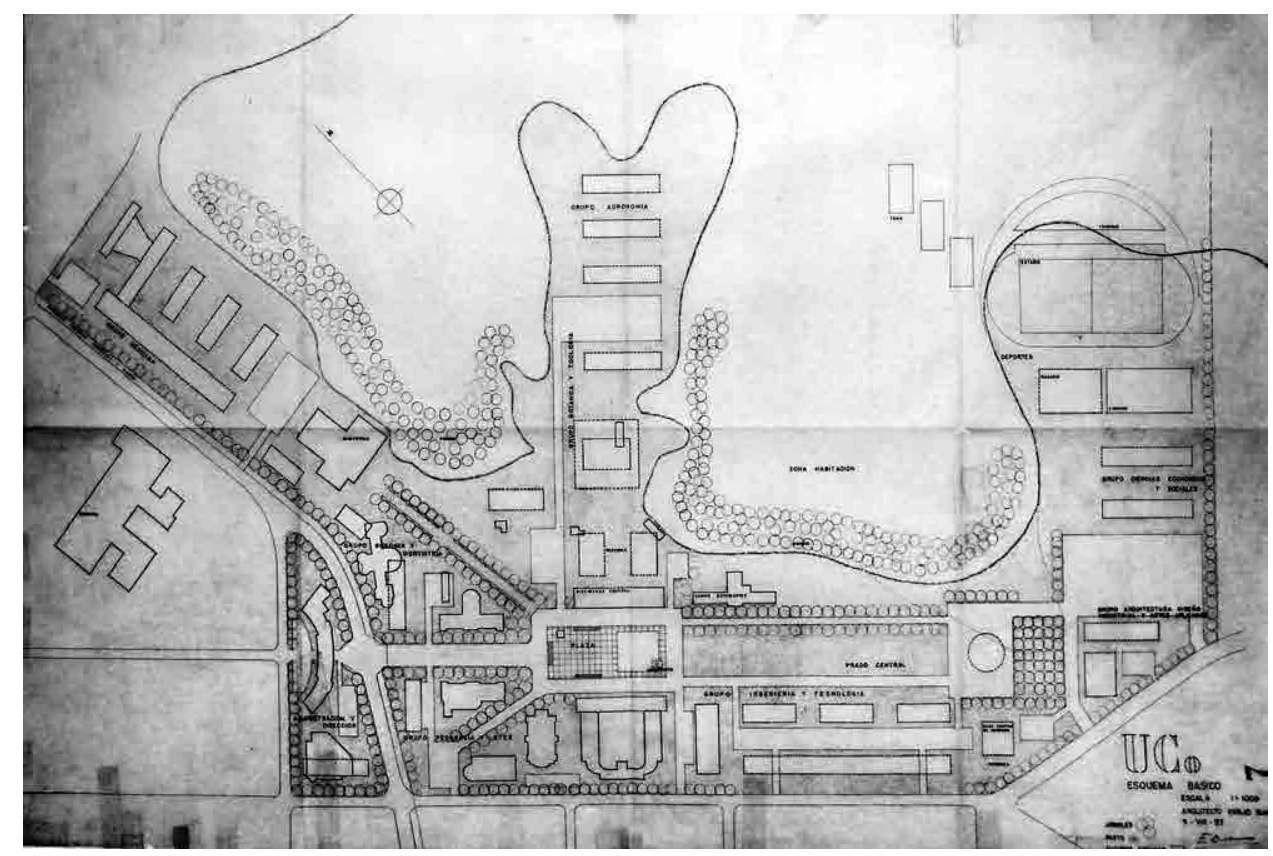

Figura 1 Plano general "Esquema básico" (agosto 1957). Fuente: Archivo de originales. FADEU, Pontificia Universidad Católica de Chile

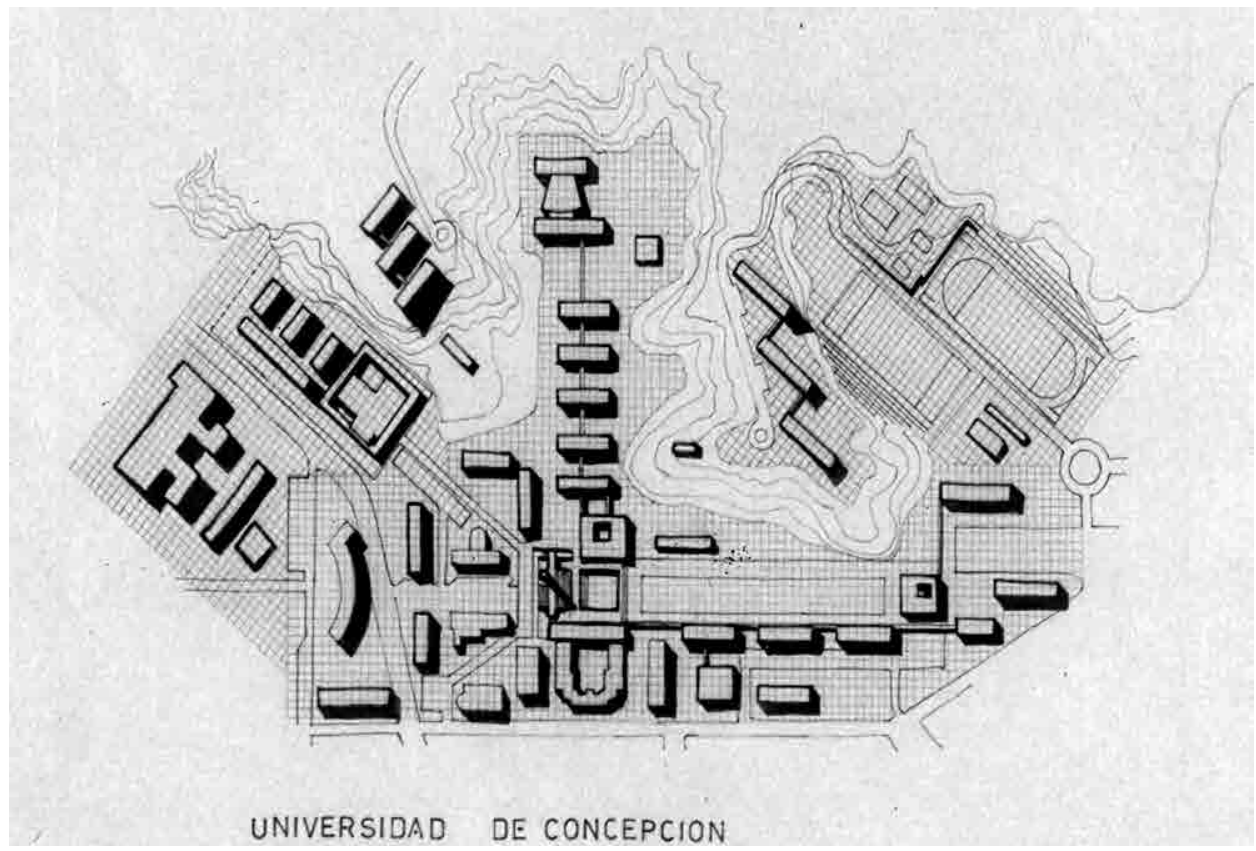

Figura 2 Re-dibujo Universidad de Concepción, trama modular. Fuente: Archivo de originales. FADEU, Pontificia Universidad Católica de Chile 
Para intentar reconstruir la génesis de las distintas etapas de proyecto de esta plaza, se digitalizaron diversos documentos originales de la fase de concepción y resultado de la propuesta urbana. La voluntad de recurrir a los archivos de originales tiene sentido en la medida que permita develar la evolución sobre una intención prístina ${ }^{5}$ de Duhart sobre esta plaza cívica y de cómo, distintos ajustes propios de la elaboración proyectual, se fueron incorporando bajo un mismo patrón: la configuración de un espacio urbano por medio de la arquitectura.

\section{MODULACIÓN COMO REGULADOR DE LA FORMA Y EL ESPACIO URBANO}

\begin{abstract}
Cuando el artista usa un método múltiple modular suele elegir una forma simple y accesible. La forma en sí misma es de importancia muy limitada; deviene en la gramática para el total de la obra. Usar formas básicas complejas sólo desbarata la unidad del todo. Usar repetidamente una forma simple reduce el campo de la obra y concentra la intensidad en la distribución de la forma. Esta distribución deviene el fin, mientras que la forma deviene el medio. (LeWitt, 1967: 81)
\end{abstract}

La primera decisión de proyecto que Emilio Duhart toma respecto a la planificación, una vez que incorpora los proyectos de arquitectura del conjunto, es la de implantar una trama modular sobre la totalidad del campus (Figura 2). Con esta herramienta proyectual, el arquitecto busca establecer un sistema sobre las decisiones del proyecto urbano y arquitectónico, conmensurando ambas escalas y fusionando sus criterios de organización.

Con la modulación superpuesta sobre la totalidad del conjunto, Duhart busca integrar las preexistencias geográficas, urbanas y arquitectónicas que anteceden su intervención con la estructura formal y espacial elaborada por él. La potencia reguladora de un sistema modular sobre la configuración de un espacio urbano, tuvo como referente contemporáneo la propuesta de Mies van der Rohe para el Instituto de Tecnología de Illinois (1939), en Chicago (Figura 3). “Mies van der Rohe planifica el campus basándose en una retícula estructural tridimensional derivada de las necesidades de un aula tipo // la unidad elegida fue un cuadrado de 7,20 metros de lado y una altura de 3,60 metros" (Carter, 2006:115). Esta trama modular le ayuda a organizar tridimensionalmente las medidas globales del conjunto $y$, con esto, a asegurar las relaciones de proporción y unidad dentro del conjunto universitario.
La modulación propuesta por Duhart para la Ciudad Universitaria de Concepción es de 7,65 metros en planta y de 3,82 metros en la altura entre pisos. Esta medida tiene como origen la modulación de base que presentaba el antiguo edificio de química, que configuraba una "U", dejando su cara abierta hacia la futura ubicación de Foro Abierto. Frente a la necesidad de aumentar la superficie de la Facultad de Química, Duhart decide adosar el nuevo edificio al existente, dando una continuidad en sus niveles de piso, con lo que además el edificio se transformaría en uno de los límites verticales más importantes de la Plaza del Foro. En la lámina de alzados y secciones del nuevo Instituto de Química (Figura 4), se puede ver que las medidas de ancho y altura se suceden entre ambos edificios, adosados así con total precisión.

En relación a las estructuras de acero, vale la pena citar a Duhart en extenso:

Sistema de Estructuras de Acero. En cuanto a la edificación, se ha propuesto y adoptado, tras múltiples consideraciones, las estructuras de acero para la mayoría de las construcciones. Esto significará un menor peso, luego una mayor economía, una gran flexibilidad en la planificación y una gran facilidad para futuras modificaciones de los edificios.

Se obtiene también una mayor claridad y homogeneidad plástica y arquitectónica. La adopción de un módulo de $7,65 \mathrm{~m}$. para la edificación, adecuado para la necesidad de la planificación y de las estructuras, hará más fácil la racionalización de la construcción y la armonía del conjunto. La planificación está regida por la trama general de 7,65 por 7,65 m, según dos cuadrículas de orientación distinta: una aproximadamente normal respecto a los puntos cardinales; la otra, según un ángulo de $40^{\circ}$ con respecto a la anterior.

Estas cuadrículas se conforman de acuerdo a las orientaciones fundamentales existentes, las que se han considerado adecuadas. Dentro de estas cuadrículas, las construcciones metálicas ofrecerán el máximo de flexibilidad de uso, de crecimiento y de adaptabilidad a los futuros cambios." (1958:10)

Las virtudes sobre el uso de esta trama modular son equivalentes a las logradas por Mies van der Rohe para el IIT, pero el resultado en la conformación espacial de ambos conjuntos son distintos. Ambos proyectos responden de forma consecuente a las condiciones propias del encargo y lugar donde se insertan, pero configurando un resultado formal y espacial que se distinguen en la experiencia espacial que ofrecen a los usuarios, influenciados de manera relevante por el contexto geográfico y urbano donde se emplazan. El hecho de modular el sitio, se transforma para Duhart en un medio eficaz para materializar su concepto de espacio urbano a gran escala; le permite prever los vectores visuales y las contenciones de aire que pretende definir mediante la distribución deliberada de los volúmenes sobre el plano, y sobre estos puestos en relación con la imponente geografía del sector (Figura 5). 


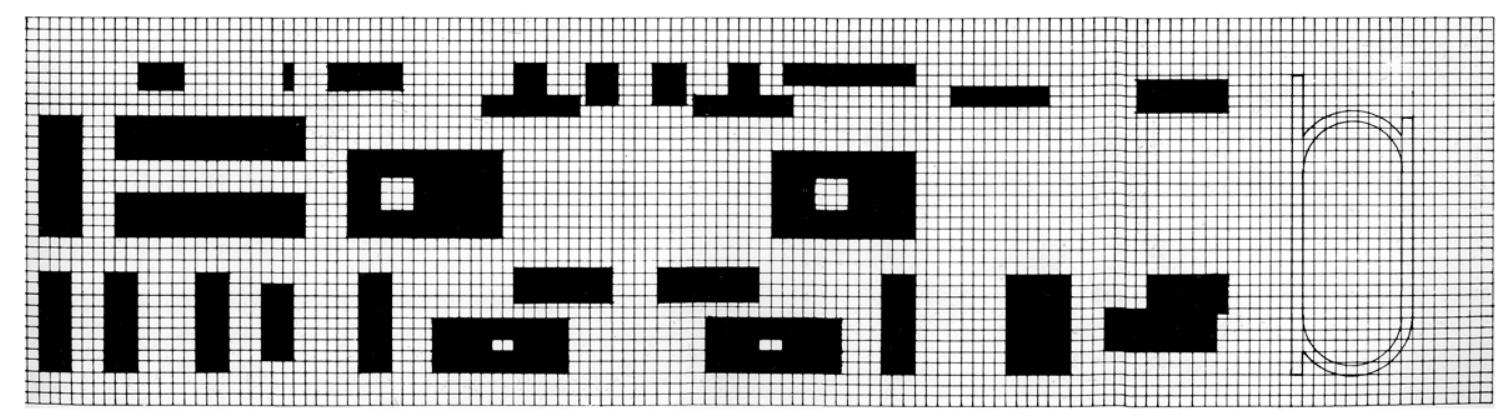

Figura 3 Trama modular Instituto de Tecnología de Illinois (1939). Mies van der Rohe. Fuente: "El arte y la ciudad contemporánea", (Benévolo, 1982:112)
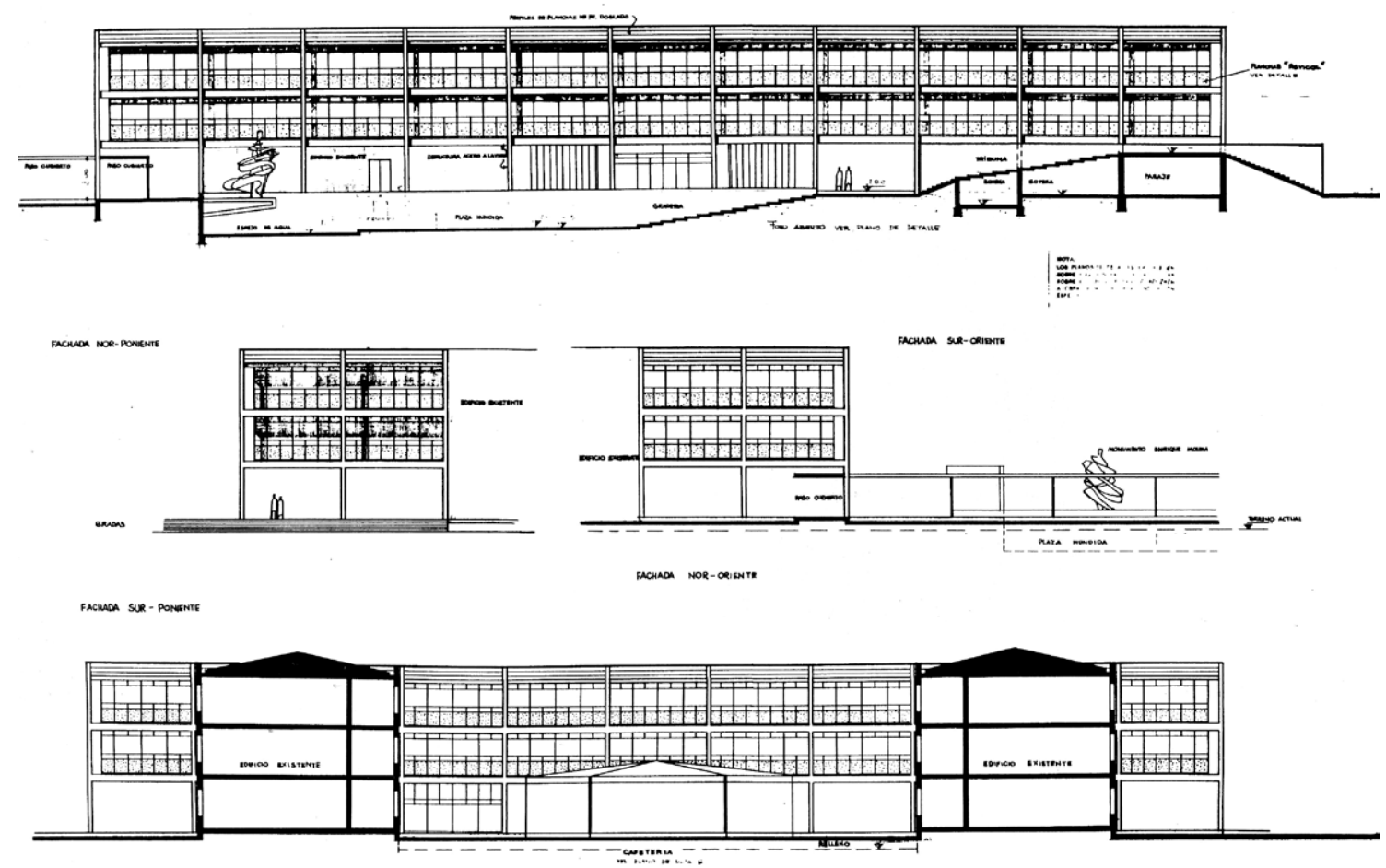

Figura 4 Elevaciones Instituto de Química (1958). Fuente: Archivo Cristián Berríos 


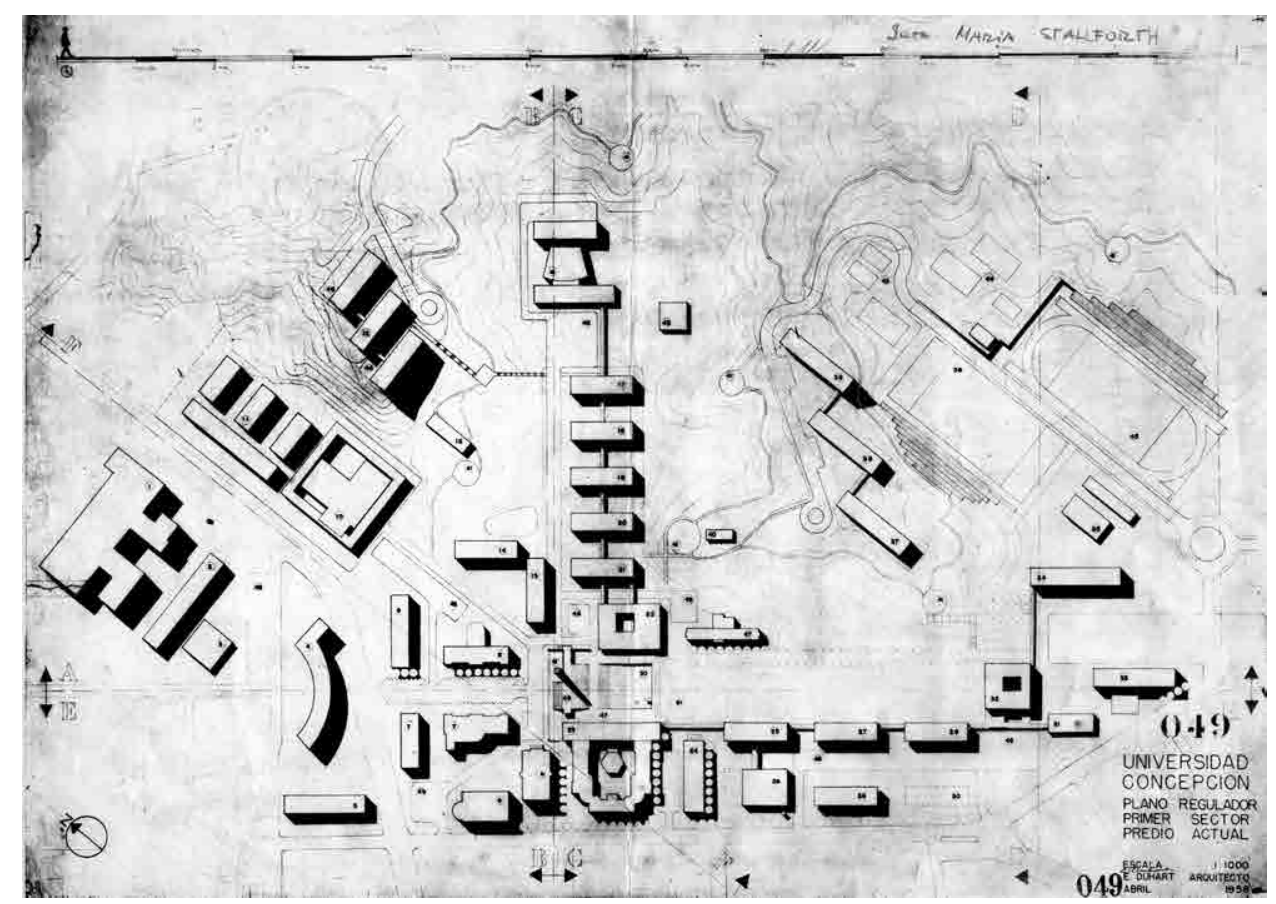

Figura 5 Plano Regulador Universidad de Concepción (1958). Fuente: Archivo Roberto Goycoolea Infante

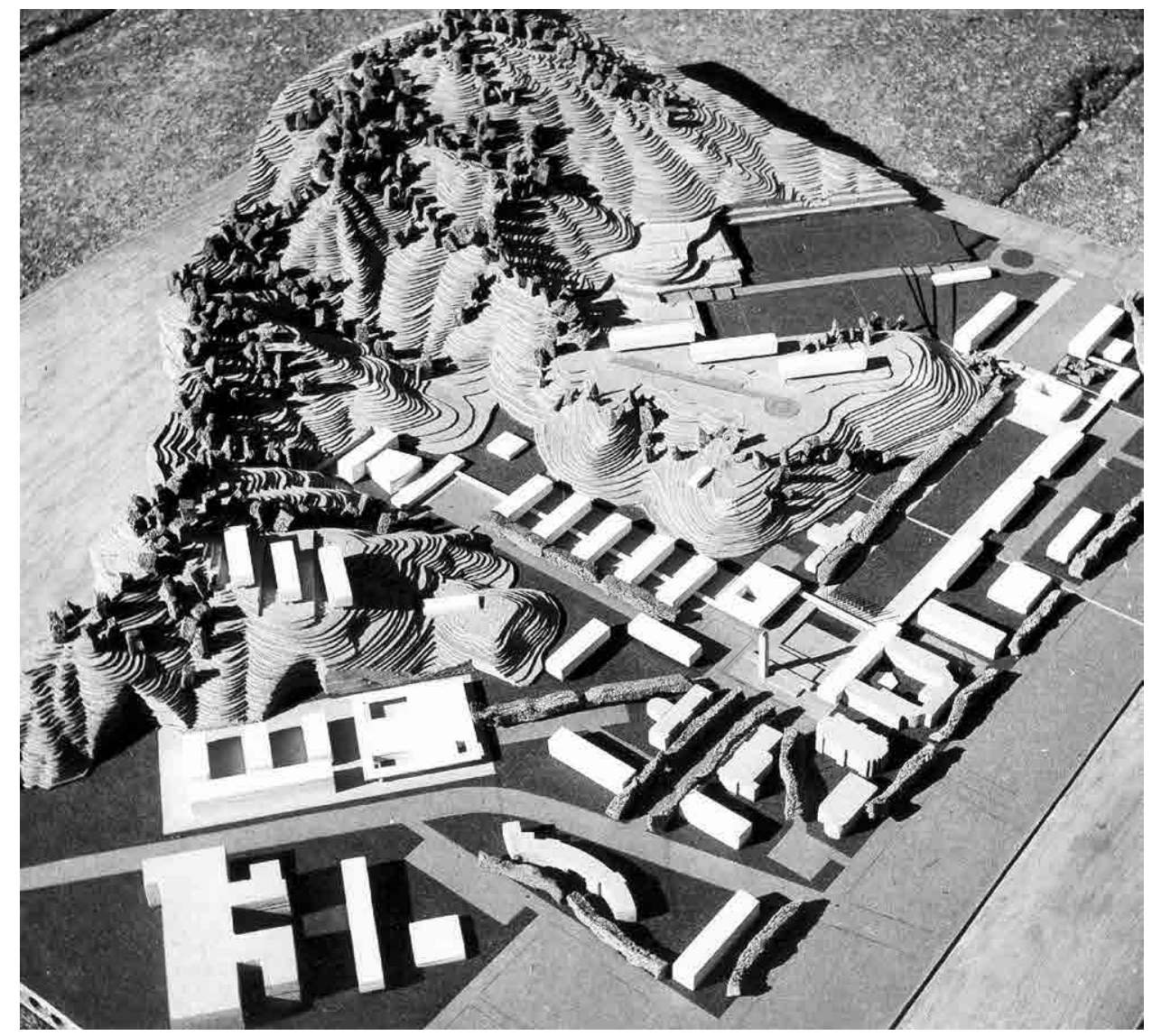

Figura 6 Fotografía Maqueta Conjunto (1958). Fuente: Archivo fotográfico Universidad de Concepción 


\section{ARQUITECTURA COMO ESTRUCTURA URBANA}

En una extensa entrevista realizada a Emilio Duhart por los arquitectos Fernando Pérez Oyarzún y Pilar Urrejola, en 1994, el arquitecto repasa gran parte de su trayectoria académica y profesional. Al momento de referirse al proyecto para la Universidad de Concepción, comparte una postura respecto a su aproximación al proyecto de arquitectura en un contexto urbano: “...quiero insertar un aspecto que es fundamental, al poco andar me di cuenta que la arquitectura sin urbanismo se queda corta como aporte del arquitecto; la forma del edificio puntual para mí ya no tenía mucho sentido"6.

Durante esta misma estadía en Chile, Duhart viaja a Concepción donde es entrevistado por el arquitecto Jaime García Molina, quien estaba redactando el libro El campus de la Universidad de Concepción: su desarrollo urbanístico y arquitectónico ${ }^{7}$. Emilio Duhart visita, entonces, el conjunto universitario recorriéndolo en toda su extensión. Cuando llegan a la Plaza del Foro Abierto, Duhart comenta: "Lo que yo quise lograr no fueron edificios con carácter, sino un conjunto con carácter"8.

Ambas citas son reveladoras a la hora de comprender la voluntad de proyecto que el arquitecto buscó desplegar para la organización del campus de la Universidad de Concepción y, en particular, en el Foro Abierto: configurar un espacio urbano mensurable, poniendo a disposición la arquitectura como artefacto capaz de dotar de estructura formal al espacio público que habita el cuerpo social (Figura 6).

La definición sobre el uso de estructuras metálicas para las edificaciones y la imposición de la retícula modular cúbica, le permite visualizar anticipadamente la composición de la arquitectura de los futuros edificios. La vinculación de medidas entre los trazados del espacio urbano y la estructura portante de los edificios, aseguran un engarce visual entre ambos aspectos del proyecto $y$, con ello, poder mesurar tanto la forma material como el vacío habitable entre ellas.

La propuesta arquitectónica para el nuevo Instituto de Química da cuenta de esta búsqueda por parte de Duhart. Tal como menciona en la misma entrevista realizada por Pérez y Urrejola, el arquitecto propone una "arquitectura impersonal", un "sistema más que una arquitectura de autor"; no es la arquitectura autoral lo que motiva a Duhart, tampoco un resultado formal innovador ensimismado, el interés es conformar un edificio que está a disposición del conjunto total, del bien común. "Una forma espacial geométricamente clara (de un espacio urbano), requiere una arquitectura muy delicada y de alta calidad. Cada error arquitectónico es inmediatamente perceptible y perjudica a la impresión total que da al espacio" (Krier, 1981:33). Duhart propone un edificio que gana consistencia formal en la expresión directa de su estructura portante, controlada por una certera composición geométrica y plástica.
La nueva construcción para el Instituto de Química obtiene su consistencia formal por medio de la expresión directa de su estructura portante, controlada por una certera composición geométrica y plástica. La longitud es de $84,5 \mathrm{~m}$. (7,65 m.x 11) y el ancho, de 15,3 m. $(7,65 \mathrm{~m} . \times 2)$; en tres niveles, con una altura total de 11,46 m. (3,82 m. x 3) (Figura 4). Los pilares cuadrados de acero $(35 \times 35 \mathrm{~cm})$, distribuidos sistemáticamente según la modulación, se empalman exterior y tangencialmente a las vigas; al dejar la lectura unitaria de los pilares en toda su altura, como elemento vertical, pauta y equilibra la tensión horizontal implícita del volumen completo. Los cerramientos del edificio retroceden $1 / 8$ del módulo $(0,95 \mathrm{~m}$.) del plomo de las vigas, con la finalidad de potenciar la esbeltez de la estructura de acero y conformar un alero por sustracción. En la planta baja, el edificio conformaba un gran pórtico que enfrentaba el Foro Abierto, conformando un espacio público cubierto y desdibujando el límite entre lo público y lo privado (Figura 7).

Esta obra sería el leitmotiv para las futuras edificaciones de la universidad: una construcción genérica, concebida desde los criterios propios de la arquitectura moderna: universalidad, economía (de medios), precisión y rigor. Este formato de edificio abstracto, le permitía a Duhart responder con una edificación flexible y atemporal tanto a los requerimientos arquitectónicos como a los urbanos, en términos del conjunto universitario.

\section{PLAZA FORO ABIERTO}

En toda su trayectoria académica y profesional, Emilio Duhart demuestra un gran interés sobre la configuración de centros cívicos en sus proyectos de gran escala: "Base Pesquera Taitao" (1941); "Ciudad Nueva Presidente Ríos" (1947); "Urbanización Achupallas" (1953); "Seminario del Gran Santiago" (1957). En cada uno de estos casos, que anteceden la planificación de la Universidad de Concepción, Duhart dedica una especial atención a detallar las plazas o centros de estas propuestas de escala urbana. Asimismo, se puede identificar en ellos recursos proyectuales que va reiterando y depurando, como: función delimitadora del espacio público de la arquitectura; polifuncionalidad de estos edificios para asegurar convocatoria de personas; utilización de pasillos cubiertos y pórticos en las plantas bajas de los edificios, como recurso para generar espacios intermedios entre lo privado y lo público, a la vez que ofrecer resguardo climático; y las dimensiones de estas plazas que se aproximan a los $50 \mathrm{~m}$ de lado, con lo que se incorpora la medida de reconocimiento del rostro y del "campo social de la visión", en palabras de Jan Gehl (2009). 


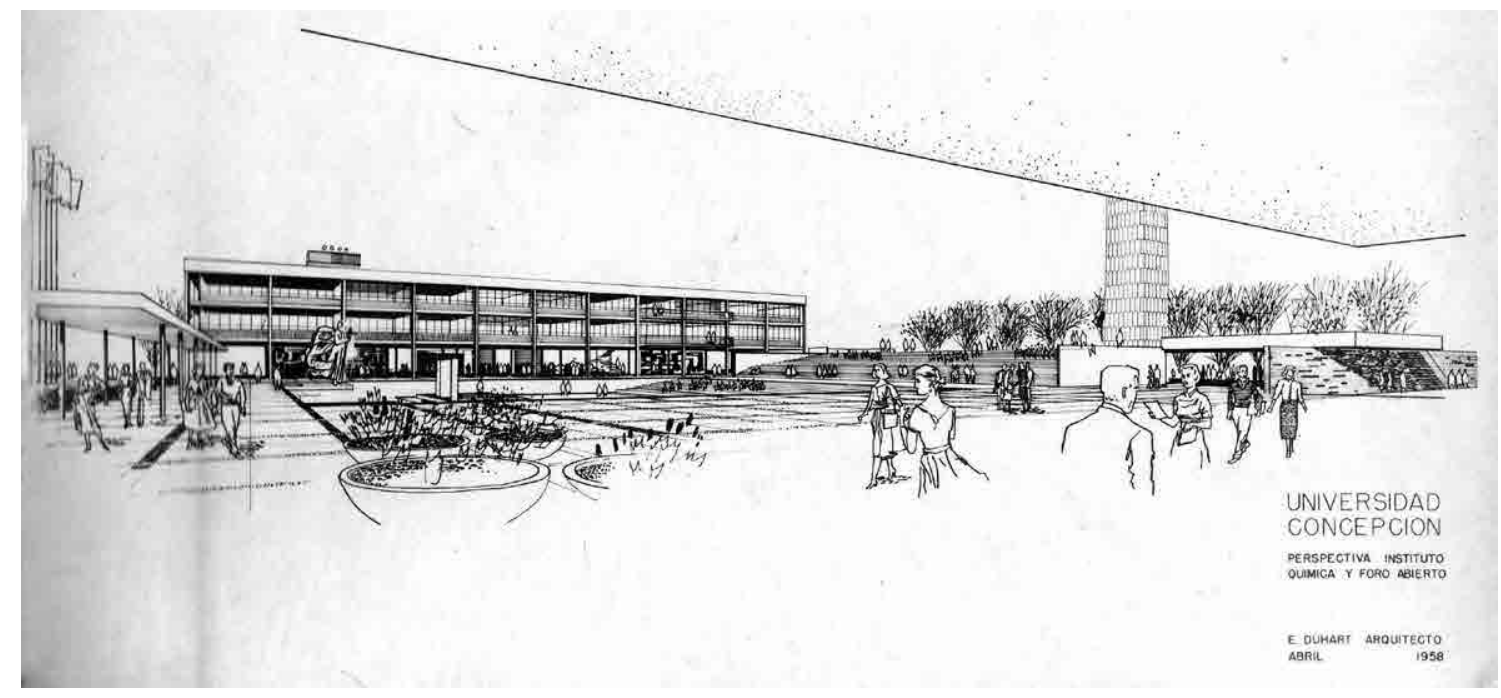

Figura 7 Perspectiva Instituto Química y Foro Abierto (1958). Fuente: Archivo Roberto Goycoolea Infante

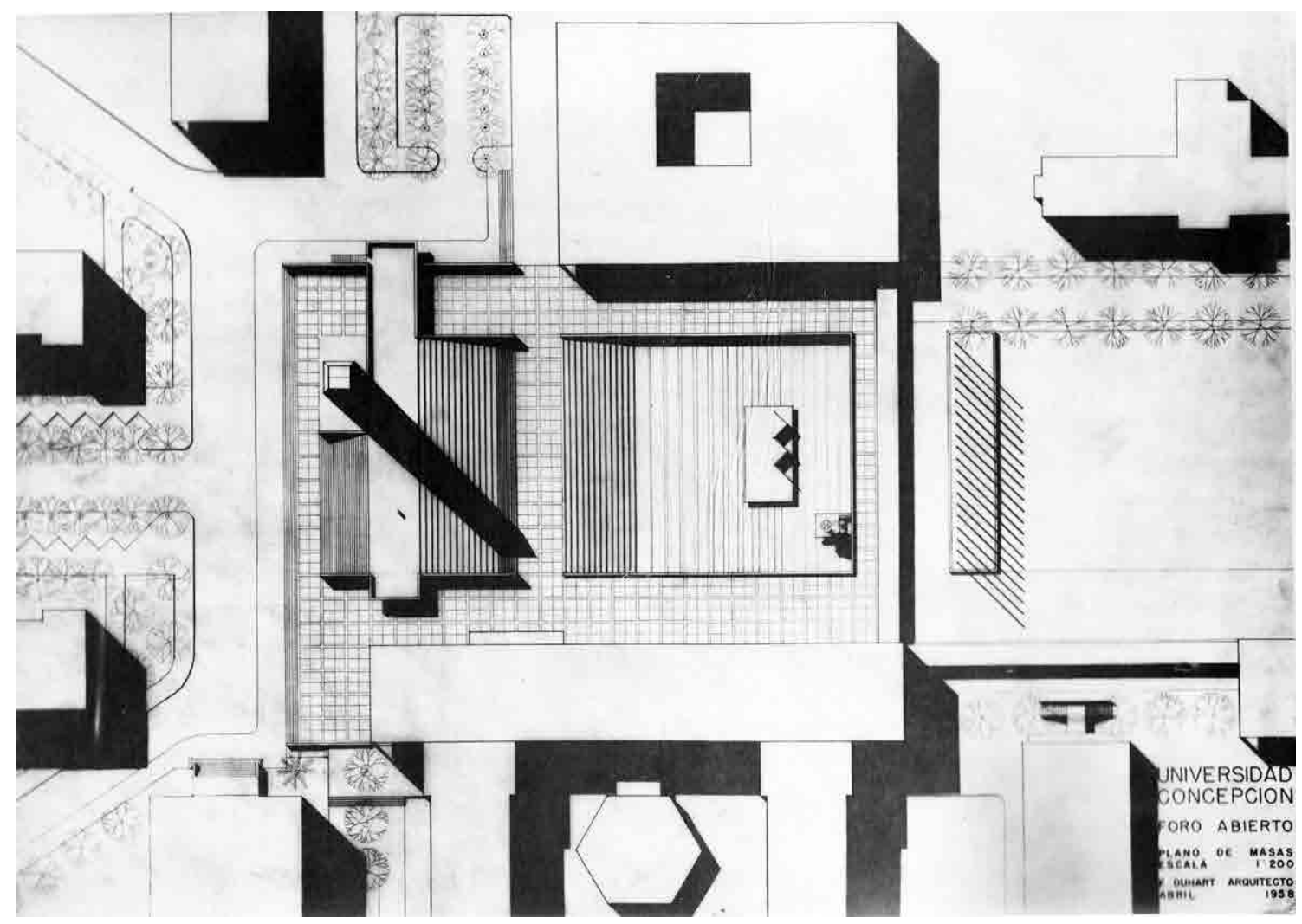

Figura 8 Plano de Masas Foro Abierto (1958). Fuente: Archivo Roberto Goycoolea Infante 
En relación al Foro de la Universidad de Concepción, Duhart señala:

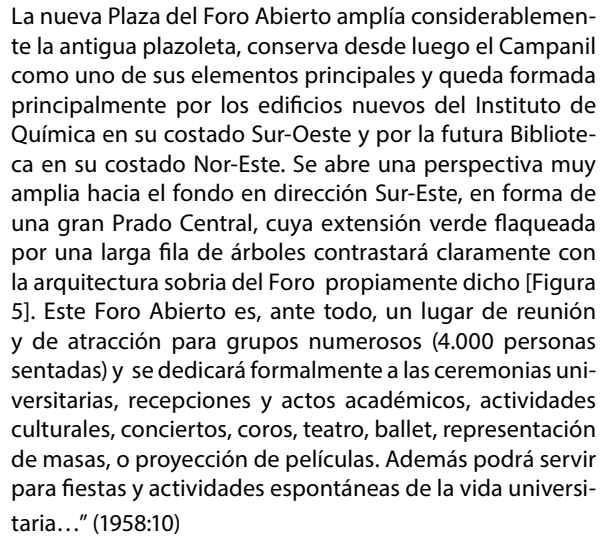
te la antigua plazoleta, conserva desde luego el Campanil como uno de sus elementos principales y queda formada principalmente por los edificios nuevos del Instituto de Química en su costado Sur-Oeste y por la futura Biblioteca en su costado Nor-Este. Se abre una perspectiva muy amplia hacia el fondo en dirección Sur-Este, en forma de una gran Prado Central, cuya extensión verde flaqueada por una larga fila de árboles contrastará claramente con la arquitectura sobria del Foro propiamente dicho [Figura 5]. Este Foro Abierto es, ante todo, un lugar de reunión y de atracción para grupos numerosos (4.000 personas sentadas) y se dedicará formalmente a las ceremonias universitarias, recepciones y actos académicos, actividades culturales, conciertos, coros, teatro, ballet, representación de masas, o proyección de películas. Además podrá servir para fiestas y actividades espontáneas de la vida universitaria..." (1958:10)

En este fragmento, el arquitecto declara la predeterminación que evidencia su voluntad sobre el concepto de plaza que concibe para el Foro Abierto; la elaboración sobre la disposición de las partes (el proyecto arquitectónico) dentro de este conjunto, como un todo, es finalmente la materialización de su visión sobre la configuración del espacio público.

El Foro Abierto es más que un perímetro de suelo liberado de construcciones; su cualidad espacial está en directa relación con los límites físicos que establecen los edificios en su contorno. Dicho esto, es correcto entender esta plaza como una agrupación de edificaciones que terminan por conformar un espacio urbano ordenado. La habilidad del arquitecto se devela en la correcta disposición de estas obras sobre el emplazamiento, en el aporte plástico de los edificios y en las relaciones formales que éstos definen (Figura 8). Si bien los bordes del pavimento del Foro Abierto conforman un rectángulo de 99,45 x 61,20 $\mathrm{m}$, los límites que establecen sobre el suelo los elementos verticales del conjunto, confinan una plaza cuadrada de $61,20 \times 61,20$ m contenida dentro de la plaza mayor.

La cara noroeste de la plaza, se configuraba por la fachada del Instituto de Química, que constituía el límite que daba la escala vertical al Foro Abierto. La estructura expuesta del edificio permitía una lectura legible y ordenada de este espacio, la total comunión de las medidas de los elementos portantes, con los despieces de pavimentos, escalinatas, etc., construían una percepción de unidad plástica de gran calidad y simpleza. La planta baja del edificio se transformaba en una gran marquesina, a modo de una stoa, que limitaban las ágoras griegas. Enfrentando este edificio, Duhart situaba la Biblioteca Central (Figura 9), de planta cuadrada con un gran patio central a modo de lucernario. El primer nivel de este edificio estaba rodeado de un pórtico en todo su perímetro, pero que doblaba su profundidad cuando enfrentaba el Foro Abierto, transformando esta marquesina también en un lugar de encuentro.

El límite hacia el noroeste está definido por la escalinata que asciende hacia la "Plaza Alta"; un suelo desplegado que adopta condiciones de elemento vertical y contiene también su función de graderías. Enfrentando esta escalinata que recorre todo el ancho del sector central de la plaza, se desciende a la "Plaza Hundida" que se direcciona hacia la fuerte presencia del "Muro del Agua" y en una mirada de contrapicado hacia la imponente "Escultura de los Fundadores". Paralelo al "Muro del Agua", sobre el suelo de mayor tránsito, el arquitecto despliega 21 mástiles que recorren el ancho del Foro Abierto. Estos mástiles configuran un límite sutil en el lado sureste de la plaza, actuando a modo de "velo", sin restringir la lectura global hacia el "Prado Central" que, a su vez, conforma la antesala con el verdadero límite que contiene al conjunto universitario: la masa de tectónica del cerro Caracol, una sección de la Cordillera de la Costa que funge también de frontera para la ciudad de Concepción.

Para Emilio Duhart, el suelo es una oportunidad de proyecto dentro del espacio urbano. Por medio de una ajustada elaboración de los niveles de pisos del Foro Abierto, el arquitecto multiplica las funciones y experiencias visuales que se puedan tener recorriendo el lugar. "La primera condición de cualquier sistema de organización formal destinada a abarcar las actividades de la vida organizada o colectiva es un plano horizontal o una serie de planos horizontales relacionados" (Martienssen, 1958:16). Duhart, entiende el suelo como proyecto, dotándolo de condiciones arquitectónicas que le permiten establecer nuevos límites y relaciones de configuración, intensificando la experiencia sobre el espacio público.

Duhart subdivide en tres niveles horizontales la Plaza del Foro Abierto. La "Plaza Principal" se eleva del nivel de suelo $1,12 \mathrm{~m}$ por medio de una escalinata de 8 huellas que recorre toda su longitud, construyendo un límite entre la "nueva" y la "antigua" universidad. Con esto fija un límite, un plano horizontal separado de la "antigua" universidad, y establece un nuevo suelo que contiene los límites de la "Plaza Principal", delimitando el nivel de mayor uso peatonal, que se vincula con los accesos del Instituto de Química y de la Biblioteca Central, asegurando sobre este nivel los encuentros entre personas.

A este nivel de la plaza se accede siempre desde sus vértices, decisión con la cual Duhart condiciona las experiencias visuales para que se realicen por medio de las perspectivas en diagonal, reconociendo siempre al menos dos de los límites verticales puestos en relación con el espacio público que definen. Al situar los recorridos cotidianos hacia los bordes de la plaza, logra también separar el tránsito masivo de las actividades que se desarrollen en la parte central del Foro Abierto, concebido para albergar distintas manifestaciones cívicas o culturales; comparten así un mismo espacio pero sin interferencias.

La "Plaza Alta", una sección del suelo elevado 3,45 m por sobre el nivel de la "Plaza Principal", funciona como "balcón". Duhart entrega al espectador una mirada de "vuelo de pájaro", permitiendo comprender la organización global del conjunto en la relación directa de los vectores visuales sobre los grandes ejes perpendiculares del conjunto universitario. Asimismo, le da fuerza a la experiencia de admirar el "Prado Central" como un gran suelo verde de escala monumental $(252,45 \times 38,25 \mathrm{~m})$. El espacio urbano limita y se contrapone con los límites de la naturaleza. Ambas realidades, lo natural y lo artificial, conviven en una concepción que las integra y potencia. Esta constatación tiene que ver también con la voluntad del arquitecto de concebir obras con un alto grado de abstracción, con el fin de establecer nuevas relaciones formales de figura y fondo con la geografía circundante, creando un nuevo paisaje. 


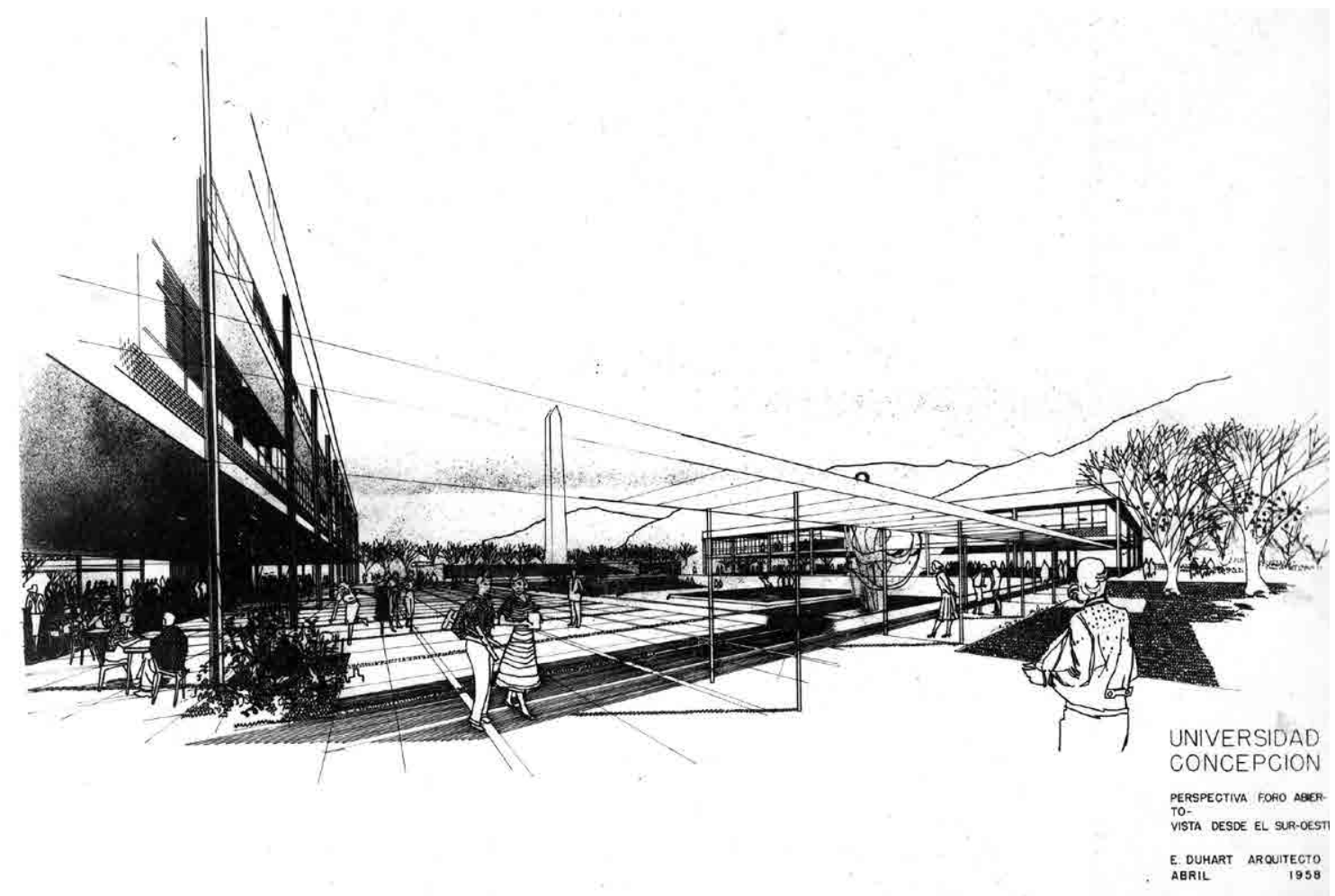

Figura 9 Perspectiva Foro Abierto (hacia la Biblioteca) (1958). Fuente: Archivo Roberto Goycoolea Infante

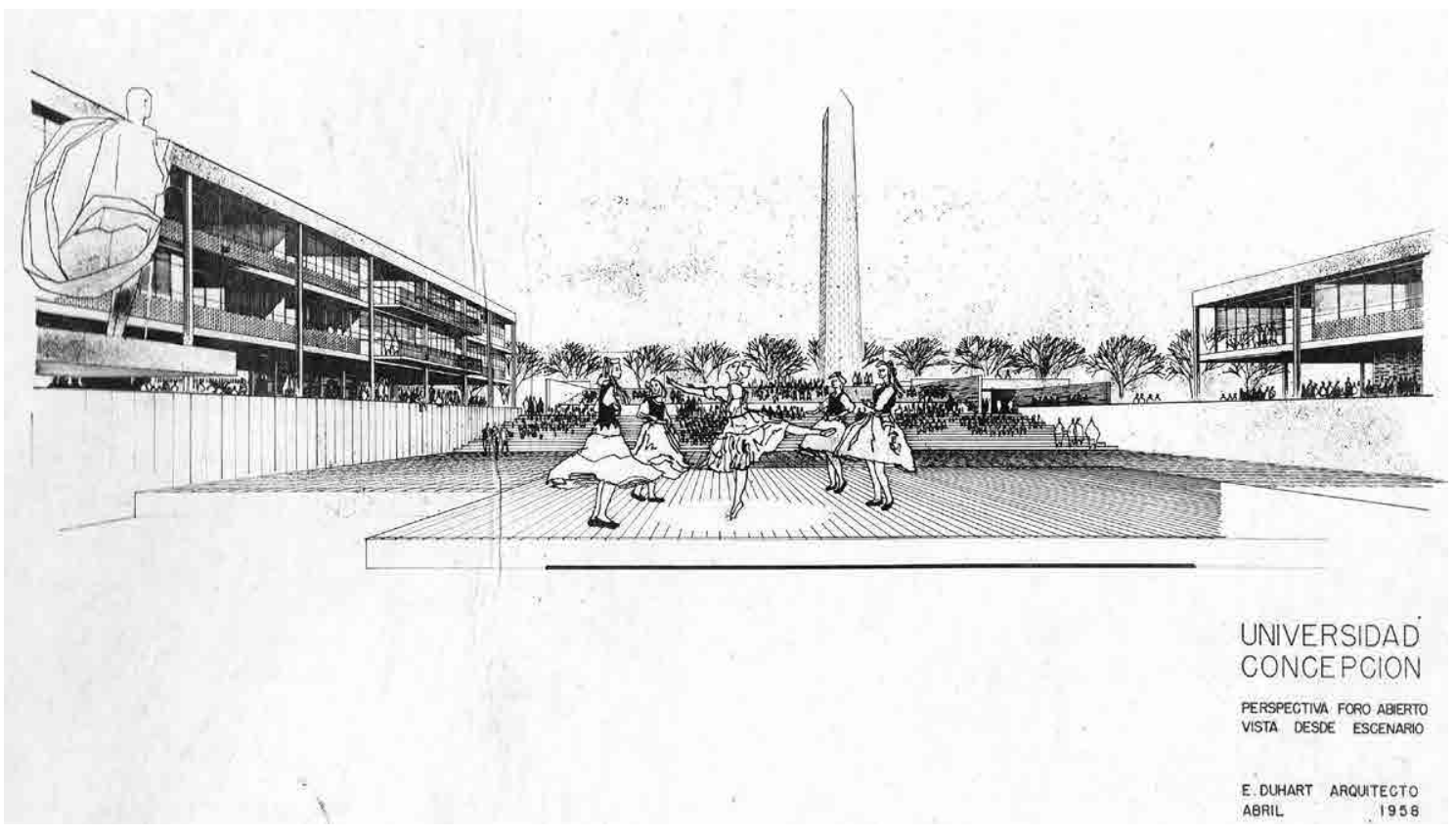

Figura 10 Perspectiva Foro Abierto desde escenario (Plaza Hundida) (1958). Fuente: Archivo Roberto Goycoolea Infante 


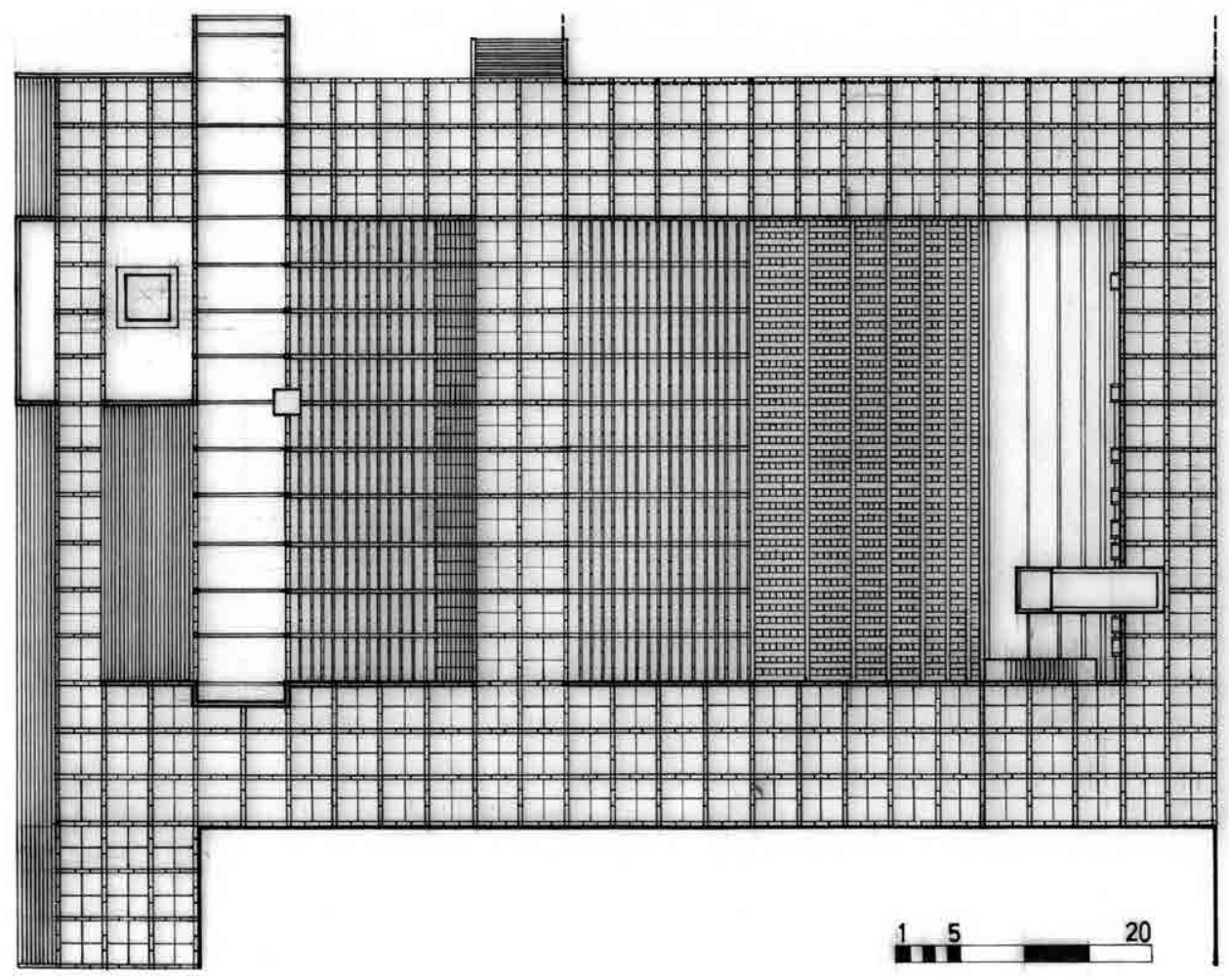

Figura 11 Propuesta desglose pavimentos Foro Abierto. Fuente: Archivo de Originales. FADEU, Pontificia Universidad Católica de Chile

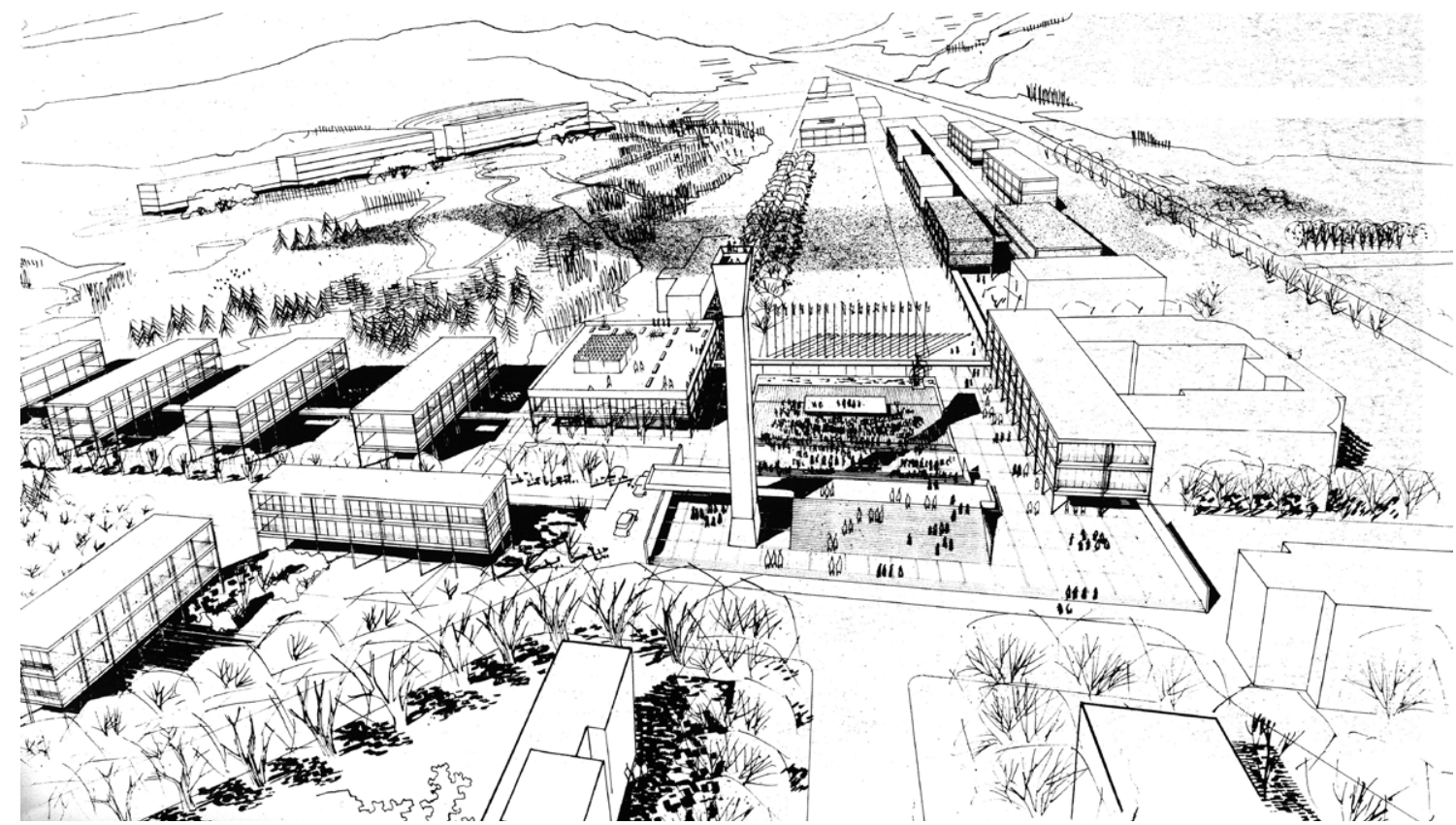


En el centro del Foro Abierto, Duhart ubica la "Plaza Hundida" (45,9 × 38,5 m), acaso el corazón del conjunto, un nuevo plano horizontal a $2,40 \mathrm{~m}$ por debajo del nivel de la "Plaza Principal" (Figura 10). Duhart dispone de una escalera de huella dilatada para descender, asumiendo su función de gradería, pero también conformando un descenso lento y pausado a un nuevo espacio separado del suelo de usos cotidianos. Aparece un nuevo lugar, más íntimo, limitado por los bordes de los muros de contención y tensionado por la ubicación excéntrica de la "Escultura de los Fundadores", que en este nivel se aprecia desde un punto de vista contrapicado. La solemnidad de la escultura se complementa con el sonido aislado del agua que cae como una cascada desde su misma base sobre un plano de agua que antecede al "Mural del Agua" y que recorre todo el ancho de este espacio. Precisamente ahí, resguardado de los recorridos cotidianos, es donde Duhart ubica un escenario móvil, reforzando así la idea de que esta plaza se transforme en un gran teatro a cielo abierto.

Por medio de una estudiada propuesta de pavimentos sobre el Foro Abierto, Duhart intenta intensificar las estructuras espaciales desarrolladas entre los distintos planos horizontales (tres niveles de la plaza) y los planos verticales (la arquitectura que limita el perímetro). Sobre el nivel principal de la plaza, el arquitecto extiende una trama continua para el pavimento, con la intención de unificar la superficie en la cual se desarrolla. Para esto, desglosa el entramado a la mitad del módulo de base, definiendo un tejido cuadrado (Figura 11). Su incidencia cobra valor en relación correlativa con los pilares de los edificios, dejando expresado de forma nítida la vinculación total de las medidas urbanas y arquitectónicas. Este calce entre pilares y el trazado del suelo refuerza la percepción de un espacio interrelacionado, de un universo de medidas al unísono, que el ojo es capáz de leer dentro de una estructura de orden, como un proceso cognitivo.

\section{CONCLUSIONES}

“Casi todo ambiente arquitectónico es una constelación muy compleja de sistemas espaciales, unos subordinados, otros coordinados, otros colindantes y otros cruzando y rodeando a los demás"

(Arnheim, 2001:16)

Emilio Duhart es parte de un grupo de arquitectos chilenos que son embajadores de la arquitectura moderna. Su formación bajo el alero de Walter Gropius en Harvard (1942) y su experiencia en el taller de Le Corbusier en París (1952), le permiten conocer de primera mano los ideales de la modernidad, pero también tomar una distancia crítica de las aproximaciones del urbanismo moderno.
En este artículo se puede reconocer que si bien el arquitecto utiliza un "lenguaje" moderno para proyectar, sus planteamientos como, cuerpo teórico, se basan en valores inmanentes de la arquitectura. La voluntad de reunir en un espacio acotado multiplicidad de edificios de usos diversos, con el fin de asegurar una mayor convocatoria de personas en la plaza del Foro Abierto, es una evidencia de una postura crítica frente a la separación de funciones pregonada por la Carta de Atenas ${ }^{9}$. De igual modo, Duhart buscar acoplar su propuesta con la organización preexistente de la universidad, dando continuidad y tensionando algunos de los ejes propuestos por el Plan de Karl Brunner (1931), pero que de su mano adoptan nuevas funciones y configuraciones sobre el espacio urbano del conjunto universitario; la idea de tabula rasa no es material de proyecto para el arquitecto.

La proposición de superponer una trama modular sobre la planificación de la universidad, permitió a Duhart sistematizar e integrar las decisiones proyectuales de la escala urbana y arquitectónica. Cada uno de los edificios propuestos respondían a sus requerimientos propios de su programa, pero también a la configuración del vacío, entendido como el espacio habitable entre los edificios; con esto supera el resultado de que lo público es el espacio residual alrededor los edificios.

Emilio Duhart proyecta una nueva dimensión de plaza pública en el Foro Abierto: por medio de la variedad de niveles que el arquitecto elabora para el suelo de esta plaza, construye una nueva geografía que multiplica las relaciones visuales sobre la experiencia del peatón. Esto le permite asimismo contener una serie de actividades poli funcionales que aseguran un permanente uso de este recinto urbano: centro de gravedad del conjunto universitario; teatro a cielo abierto; rótula de la antigua y nueva universidad; símbolo universitario y también ciudadano, como una gran ejemplo de espacio público democrático e integrador que trasciende su vocación universitaria, instalándose como uno de los "lugares" de mayor sentido urbano de Concepción.

Duhart no dejó textos sobre su teoría arquitectónica y urbana, de manera que para intentar develarla se debe hacer un ejercicio de "lectura entre líneas" de sus proyectos y obras. A través de esa mirada, se puede asegurar que el Plan Director para la Universidad de Concepción constituye una buena síntesis de sus permanentes cavilaciones sobre el espacio urbano ${ }^{10}$. 


\section{REFERENCIAS BIBLIOGRÁFICAS}

ARNHEIM, Rudolf. La forma visual de la arquitectura. Barcelona: Ed. Gustavo Gili, 2001.

BERRÍOS, Cristián. Emilio Duhart: Ciudad Universitaria de Concepción. Elaboración de un espacio urbano moderno. Tesis doctoral inédita, Universidad Politécnica de Cataluña-Barcelona, 2012

BENÉVOLO, Leonardo. Diseño de la Ciudad V.5. El arte y la ciudad contemporánea. Barcelona: Ed. Gustavo Gili, 1982.

CARTER, Peter. Mies van der Rohe trabajando. Londres: Ed. Phaidon Press, 2006.

DUHART, Emilio. Extracto Memoria del Plan Director para la Universidad de Concepción, Boletín Informativo Universidad de Concepción, n 6, julio 1958, p 10-12

GEHL, Jan. La humanización del espacio urbano. BarceIona: Ed. Revertré, 2009 (reimpresión).

KRIER, Rob. El espacio urbano. Barcelona: Ed. Gustavo Gili, 1981.

LEWITT, Sol. Paragraphs on Conceptual Art. Artforum 5, $\mathrm{n}^{\circ} 10$, julio 1967, pp. $79-83$

MARTIENSSEN, Rex. La idea del espacio en la arquitectura griega. Buenos Aires: Ed. Nueva Visión. 1977 